\title{
Coronary artery calcium in primary prevention
}

CPD

Alexander Chua, Ron Blankstein, Brian Ko

\section{Background}

Identifying high-risk asymptomatic individuals remains the cornerstone of cardiovascular disease prevention. Coronary artery calcium is a highly specific marker of atherosclerosis that can be quantified using non-contrast computed tomography. The resulting calcium score has the capacity to improve current methods of risk stratification.

\section{Objective}

The aim of this article is to provide an overview of calcium scoring, including its method of acquisition, indications, interpretation and role in prognostication.

\section{Discussion}

Calcium score has been shown to convincingly predict future cardiovascular risk in the asymptomatic population across a wide range of ethnicities, ages and sexes. Individuals at intermediate Framingham risk benefit the most from calcium scoring, which can be used to inform the need for preventive pharmacotherapy. Calcium scoring can be repeated after five years to reassess cardiovascular risk, especially when there is a decision to defer statin therapy on the basis of absence of coronary calcium.
CORONARY ARTERY DISEASE (CAD) is the single leading cause of disease morbidity and mortality in Australia and is responsible for approximately in one in 10 deaths. ${ }^{1}$ While chronic angina is a common presentation of CAD, up to half of individuals will initially present with myocardial infarction or sudden cardiac death. ${ }^{2}$ For the purposes of prevention, it is necessary to identify asymptomatic at-risk individuals likely to benefit from the early detection and treatment of CAD.

In Australia, risk assessment is recommended to be performed using the National Vascular Disease Prevention Alliance tool, which is in turn based on the Framingham Risk Equation. ${ }^{3}$ These office-based calculators integrate several risk factors in order to estimate absolute cardiovascular risk. ${ }^{3,4}$ While these tools offer a quick and cost-effective method of risk assessment, it is recognised that many patients are not identified as high risk prior to their first coronary event. ${ }^{5,6}$

Coronary artery calcium (CAC) is a highly specific marker of atherosclerosis that has prognostic value in predicting cardiovascular risk. ${ }^{3,7}$ The quantification of coronary calcium by non-contrast computed tomography (CT) has emerged as an attractive tool to improve risk stratification and risk reclassification in asymptomatic individuals.
What is coronary artery calcium? CAC refers to calcium deposits in the coronary arteries that occur during atherosclerotic plaque formation. ${ }^{8}$ Previously thought to be a result of ageing, coronary calcium is an abnormal finding that can be identified as early as the second decade of life following fatty streak formation. ${ }^{9}$

The link between coronary calcium and atherosclerosis has been known since the 1970 s, when fluoroscopically detected calcium was associated with adverse cardiovascular events. ${ }^{10}$ With the development of CT imaging, coronary calcium was found to correlate with atherosclerotic plaque volumes both in pathological specimens and in vivo using intravascular ultrasonography. ${ }^{11,12}$ As a surrogate measure of atherosclerotic burden, calcium score can be considered a reflection of an individual's cumulative risk factor exposure across their lifetime.

How is calcium scoring performed? Calcium scoring involves a CT scan of the heart using a slice thickness of $3 \mathrm{~mm} .{ }^{3}$ The study is complete within 10 minutes, and the radiation dose is low at approximately $1 \mathrm{mSv}{ }^{13}$ The scan is performed without contrast administration and therefore does not provide luminal stenosis 
assessment. This is unlike coronary CT angiography, which is typically performed for symptomatic patients with suspected obstructive CAD. ${ }^{7}$

CAC appears on CT as $>1 \mathrm{~mm}^{2}$ areas of hyperattenuation ( $>130$ Hounsfield units) and is most commonly quantified using the Agatston method. ${ }^{13,14}$ In this method, the angiographer reviews each $3 \mathrm{~mm}$ thick CT slice of the heart (usually $12-16 \mathrm{~cm}$ in length) to identify coronary calcium. ${ }^{13}$ The amalgamated volume and density of coronary calcium is then calculated using dedicated computer software (Figure 1$).^{3}$

\section{How are calcium scores interpreted?}

Calcium scores are represented by an absolute numerical value and a percentile based on sex, age and ethnicity. ${ }^{7}$ The Cardiac Society of Australia and New
Zealand (CSANZ) divides calcium scores into the following groups on the basis of absolute values: $\mathrm{CAC}=0, \mathrm{CAC}=1-100$, $\mathrm{CAC}=101-400$, and $\mathrm{CAC}>400$, with each group corresponding to a particular level of cardiovascular risk (Table 1$).^{3}$

\section{What are the benefits of calcium scoring? \\ Independent prediction of cardiovascular risk}

Large prospective studies of asymptomatic individuals have shown that calcium score predicts important cardiovascular outcomes, including coronary events, myocardial infarction and all-cause mortality. ${ }^{15-17}$ Initial evidence supporting the prognostic value of coronary calcium was provided by the landmark Multiethnic Study of Atherosclerosis (MESA), a prospective cohort of 6814 individuals

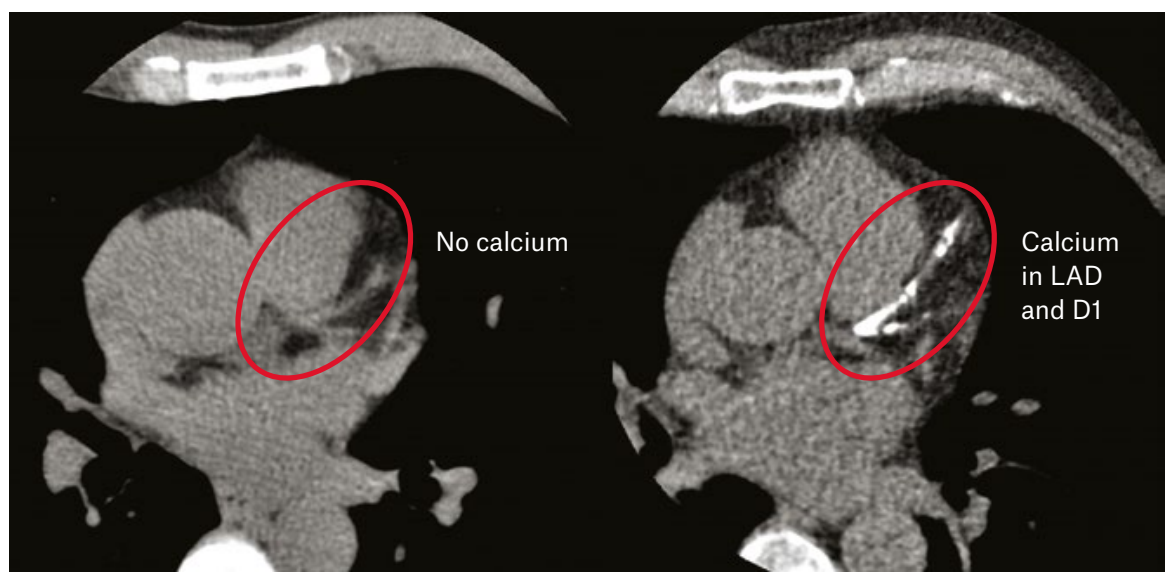

Figure 1. Coronary artery calcium in the left anterior descending (LAD) and first diagonal (D1) arteries

Table 1. Interpretation of coronary calcium score ${ }^{3}$

\begin{tabular}{llr}
\hline Calcium score & Interpretation & $\begin{array}{r}\text { Risk of myocardial } \\
\text { infarction/stroke at 10 years }\end{array}$ \\
\hline 0 & Very low risk & $<1 \%$ \\
\hline $1-100$ & Low risk & $<10 \%$ \\
\hline $101-400$ & Moderate risk & $10-20 \%$ \\
\hline $101-400$ and $>75$ th percentile & Moderately high risk & $15-20 \%$ \\
\hline$>400$ & High risk & $>20 \%$ \\
\hline
\end{tabular}

from four major ethnic groups. ${ }^{15}$ This study found an independent and graded association between calcium score and adverse coronary events over 3.8 years of follow-up. ${ }^{15}$ Subsequent studies have validated calcium scoring in a wide range of ages, sexes and clinical risk factor burdens, ${ }^{18-20}$ and have confirmed its long-term prognostic value over $>10$ years of prospective follow-up. ${ }^{16,17}$

\section{Utility as a negative risk marker}

A key advantage of calcium scoring is that while high scores are associated with elevated cardiovascular risk, the absence of coronary calcium is a negative risk marker that confers a favourable prognosis. ${ }^{3}$ In a meta-analysis of 71,595 asymptomatic individuals, the cardiovascular event rate in those with $\mathrm{CAC}=0$ was $0.47 \%$ over 50 months of follow-up, compared with $4.14 \%$ in those with a positive calcium score. ${ }^{21}$ Studies assessing all-cause mortality have reported similarly low event rates of $<0.1 \%$ annually. ${ }^{22,23}$

The negative predictive value of zero coronary calcium appears to be greatest in individuals at intermediate risk by traditional risk calculators; $45 \%$ of these patients will have $\mathrm{CAC}=0$, placing them at a low cardiovascular risk and removing the need for preventive therapy such as statins. ${ }^{24}$ Conversely, the power of zero coronary calcium is limited in high-risk individuals, who remain at greater than low cardiovascular risk despite $\mathrm{CAC}=0 .{ }^{24}$

\section{Improvement on traditional risk calculators}

Studies have shown that the addition of calcium scoring to traditional risk calculators improves the accuracy of risk prediction. ${ }^{15,25,26}$ In particular, the use of calcium scoring results in a significant net reclassification improvement (NRI), defined as the proportion of a population correctly reassigned to a higher or lower risk class following calcium scoring. Data from MESA determined that calcium scoring produced an NRI of $25 \%$ when added to the Framingham Risk Equation, and that this improvement was greatest in the intermediate-risk population, for whom the NRI was $55 \% .{ }^{25}$ These findings have been corroborated by subsequent studies. ${ }^{3,26,27}$ 
What are the limitations of calcium scoring?

High-risk and low-risk asymptomatic patients

While intermediate-risk patients benefit the most from calcium scoring, studies have generally reported smaller reclassification benefits among low- and high-risk individuals. ${ }^{25-27}$ In a study of 2028 patients, the NRI of calcium scoring when added to the Framingham Risk Equation was $12 \%$ in the low-risk group and $34 \%$ in the high-risk group, compared with $52 \%$ in the intermediate risk group. ${ }^{27}$

The smaller reclassification benefit in low-risk populations can be attributed to a high prevalence of zero coronary calcium, which results in many patients remaining at low risk following calcium scoring. ${ }^{28}$ Conversely, the low prevalence and reduced negative predictive value of $\mathrm{CAC}=0$ in high-risk individuals limits the ability of calcium scoring to reclassify cardiovascular risk in this population. ${ }^{24}$

\section{Symptomatic patients}

Calcium scoring is not currently recommended for symptomatic patients by the CSANZ. ${ }^{3}$ Instead, guidelines recommend the upfront use of stress testing or cardiac $\mathrm{CT}$ angiography, which provide assessment of ischaemia and luminal stenosis, respectively. ${ }^{3}$ While calcium scoring is a reliable measure of atherosclerotic burden, it does not distinguish between obstructive and non-obstructive CAD. ${ }^{29}$

\section{Non-calcified plaque}

Calcium scoring does not provide information on the presence or burden of non-calcified plaque, which has low radiological attenuation and requires contrast administration to be assessed. ${ }^{30}$ This is typically performed using coronary CT angiography. ${ }^{30}$ Non-calcified plaque burden has been identified as an important predictor of future adverse cardiac events, particularly among symptomatic patients. ${ }^{31}$ However, in asymptomatic patients, the use of coronary $\mathrm{CT}$ angiography to assess non-calcified plaque has not been shown to improve cardiovascular risk assessment when added to calcium scoring. ${ }^{32,33}$

\section{Cost effectiveness}

There are no available Australian data assessing the cost effectiveness of calcium scoring. ${ }^{3}$ Hence, the impact of calcium scoring on downstream investigations, procedures and resource use is not known. As no Medicare Benefits Schedule items exist for calcium scoring at this time, the cost of the test is funded by patients, approximately $\$ 150-200$ per scan.

\section{Other considerations}

Calcium scoring involves approximately $1 \mathrm{mSV}$ of radiation exposure, which is comparable to two screening mammographies. ${ }^{13}$ The benefit of improved cardiovascular risk assessment must be considered against the risks of radiation exposure (eg malignancy).

Calcium scoring is performed with a CT scan of the chest and may result in incidental findings. In a study of 966 individuals who underwent calcium scoring, $8.2 \%$ of patients had incidental findings requiring further investigation or treatment. ${ }^{34}$ Incidental findings may result in increased healthcare resource expenditure and psychological harm. ${ }^{34}$

\section{Who should undergo calcium scoring?}

The CSANZ recommends the use of the Framingham Risk Score to select patients for calcium scoring, as studies assessing its predictive value have typically used the Framingham Risk Equation as a comparator. $^{3}$

The CSANZ suggests that calcium scoring is appropriate for asymptomatic patients at intermediate Framingham risk, for whom there is strong evidence supporting its ability to improve cardiovascular risk assessment. ${ }^{3}$ Calcium scoring may also be appropriate for lower-risk patients (6-10\% 10-year risk) with concerning features not considered by the Framingham Risk Equation. The CSANZ suggests that in this group, Aboriginal and Torres Strait Islander patients and patients with a strong family history of premature coronary heart disease or diabetes may derive benefit from the increased accuracy of calcium scoring. ${ }^{3}$

\section{Who should not undergo calcium scoring?}

The CSANZ recommends against calcium scoring for high-risk and very low-risk patients ( $<6 \% 10$-year risk), for whom calcium scoring has a limited ability to reclassify cardiovascular risk. ${ }^{3}$ The use of calcium scoring for symptomatic patients is also not recommended because of insufficient evidence supporting its use over functional testing, CT angiography and invasive coronary angiography (Figure 2). ${ }^{3}$

\section{How does calcium score guide management?}

As calcium scoring improves cardiovascular risk assessment, it has the capacity to inform the risk-based selection of patients for preventive pharmacotherapy. A number of large observational studies show that calcium score identifies patients who may benefit from aspirin and statin therapy. ${ }^{35-37}$

\section{Aspirin}

Aspirin is not generally recommended for primary prevention as its cardiovascular benefits are outweighed by an increased risk of bleeding. ${ }^{3}$ However, calcium scoring has been shown to identify a subgroup of patients for whom prophylactic aspirin may be beneficial.

Using data from MESA, Miedema et al showed that aspirin had a net benefit among individuals with CAC $>100 .{ }^{35}$ In this group, the calculated five-year number needed to treat (NNT) of aspirin to prevent one coronary event was significantly less than the number needed to harm $(\mathrm{NNH})$ for major bleeding. ${ }^{35}$ Conversely, aspirin showed no net benefit for individuals with $\mathrm{CAC}=0$ and could not be universally recommended for those with CAC $=1-99 .{ }^{35}$

\section{Statins}

Studies have also assessed the ability of calcium scoring to guide lipid-lowering therapy. In a study of 950 individuals, the calculated five-year NNT of statins to prevent one coronary event was 549 for individuals with $\mathrm{CAC}=0$, compared with 24 for those with $C A C>100$, suggesting 
that statins should be prescribed for individuals with $\mathrm{CAC}>100$ but may be withheld for those with $\mathrm{CAC}=0 .{ }^{36}$ These findings were recently corroborated in a large study of 13,644 individuals over 9.4 years of follow-up. ${ }^{37}$

\section{Recommendations}

On the basis of the available evidence, the CSANZ suggests preventive therapy with aspirin and a statin is appropriate for individuals with CAC $>100$ and recommends no therapy for individuals with $\mathrm{CAC}=0 .{ }^{3}$ For individuals with $\mathrm{CAC}=1-99$, preventive therapy can be considered if concerning features are present (eg family history of premature heart disease).$^{3}$ Lifestyle management and risk factor control should be implemented for all patients regardless of calcium score (Figure 3).

\section{Should calcium scoring be repeated?}

CAC either increases or remains stable over time and does not regress. ${ }^{38}$ Calcium scoring can be repeated in order to reassess cardiovascular risk, provided that this would lead to changes in patient management.
For this reason, the role of repeat calcium scoring is likely greatest for individuals with $C A C=0$ for whom statins have been withheld. In this group, the CSANZ suggests repeat calcium scoring may be considered after five years, on the basis of data showing a mean time of conversion to a positive score of 4.1 years. ${ }^{3,39}$

Conversely, patients with CAC $>100$ should not undergo repeat scoring as they are already candidates for preventive therapy. ${ }^{3}$ Repeat calcium scoring may be less useful following statin therapy because statins mildly increase coronary calcium as a consequence of plaque stabilisation. ${ }^{40}$

\section{Who should undergo calcium scoring?}

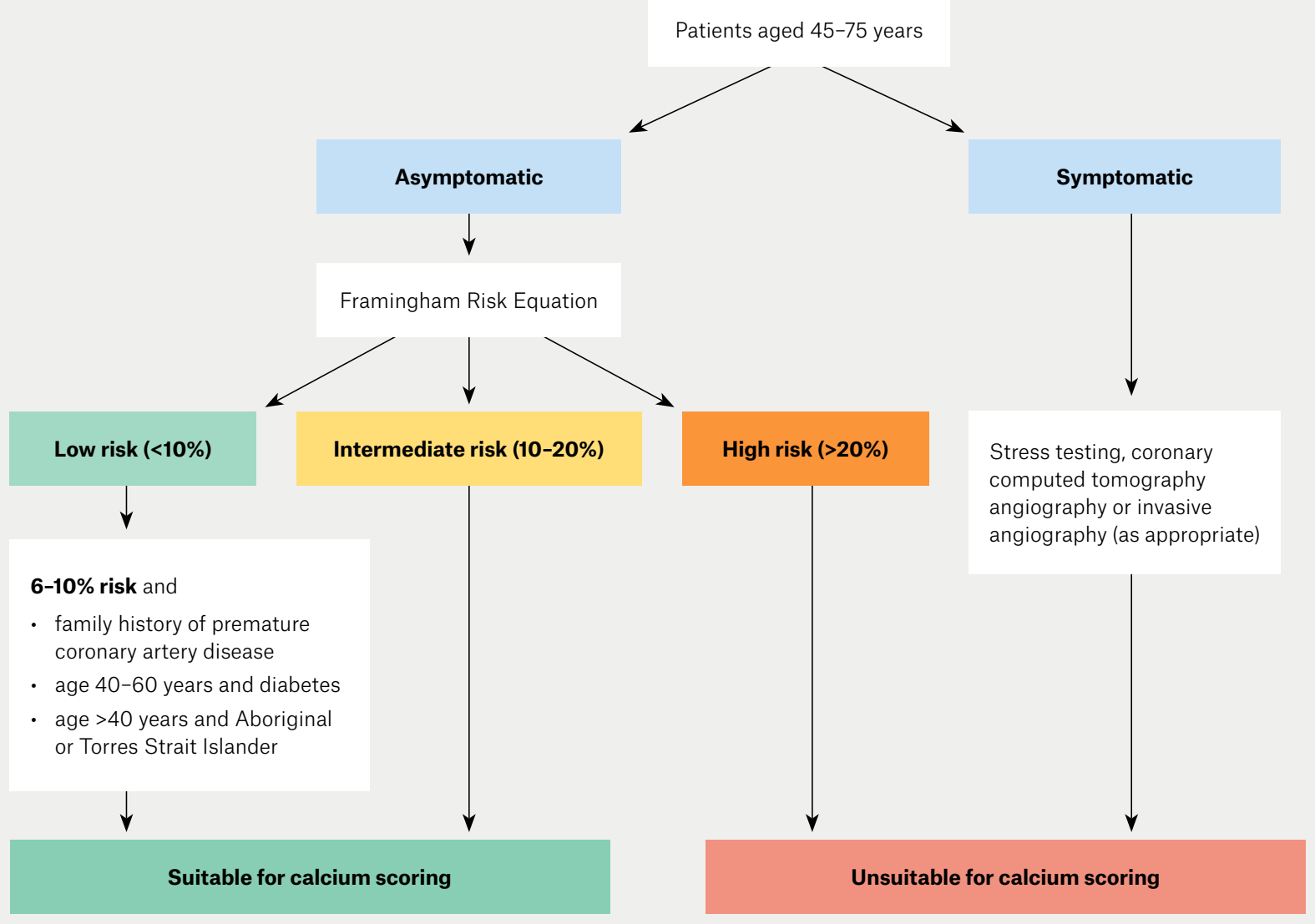

Figure 2. The Cardiac Society of Australia and New Zealand's recommended indications for coronary artery calcium scoring ${ }^{3}$ 


\begin{tabular}{|c|c|c|c|c|}
\hline & & $C A C=0$ & $C A C=1-100$ & CAC $>100$ \\
\hline Population (\% patients) ${ }^{35}$ & & $56 \%$ & $26 \%$ & $18 \%$ \\
\hline Annual coronary event rate ${ }^{36}$ & & $0.1 \%$ & $0.5 \%$ & $1.9 \%$ \\
\hline Annual cardiovascular event rate ${ }^{35}$ & & $0.4 \%$ & $0.8 \%$ & $2.4 \%$ \\
\hline \multicolumn{5}{|c|}{ NNT to prevent one coronary event over five years } \\
\hline NNT aspirin ${ }^{35}$ & FRS $<10 \%$ & 2,036 & 571 & 173 \\
\hline $\mathrm{NNH}=442$ for major bleeding & FRS $\geq 10 \%$ & 808 & 146 & 92 \\
\hline \multirow[t]{4}{*}{ NNT statin ${ }^{36}$} & & 549 & 94 & 24 \\
\hline & & $\downarrow$ & $\downarrow$ & $\downarrow$ \\
\hline & & Reassure & $\begin{array}{l}\text { Individualise statin } \\
+ \text { aspirin }\end{array}$ & $\begin{array}{l}\text { Recommend statin } \\
\text { + aspirin }\end{array}$ \\
\hline & & All pa & yle management and & tor control \\
\hline
\end{tabular}

Figure 3. Treatment implications of coronary artery calcium (CAC) score ${ }^{41}$

FRS, Framingham Risk Score; NNH, number needed to heal; NNT, number needed to treat

Adapted with permission from the British Institute of Radiology

\section{Summary}

Coronary calcium score is a surrogate marker of calcified atherosclerotic burden that independently predicts cardiovascular risk and mortality. It is a widely available test typically performed in intermediaterisk asymptomatic patients. Risk estimates provided by calcium scoring improves the accuracy of traditional risk calculators and can be used for the risk-based selection of patients for preventive pharmacotherapy.

\section{Authors}

Alexander Chua MBBS (Hons)

Ron Blankstein MD, President, Society of

Cardiovascular Computed Tomography; Director,

Cardiac Computed Tomography, Brigham and

Women's Hospital, Boston, MA

Brian Ko MBBS (Hons), MD, PhD, Interventional Cardiologist and Cardiac CT Specialist,

MonashHeart, Monash Medical Centre, Monash University, Vic. brianshiuhangko@gmail.com
Competing interests: RB has received research support from Amgen Inc and Astellas Inc, outside the submitted work. BK has received research funding from Canon Medical, and honoraria from Abbott, Bristol-Myers Squibb, Novartis, GlaxoSmithKline and Medtronic, outside the submitted work.

Funding: None

Provenance and peer review: Not commissioned, externally peer reviewed.

\section{References}

1. Australian Institute of Health and Welfare. Australia's health 2018. Australia's health series no. 16. AUS 221. Canberra, ACT: AlHW, 2018.

2. Lerner DJ, Kannel WB. Patterns of coronary heart disease morbidity and mortality in the sexes: A 26-year follow-up of the Framingham population. Am Heart J 1986;111(2):383-90. doi: 10.1016/0002-8703(86)90155-9.

3. Liew G, Chow C, van Pelt N, et al. Cardiac Society of Australia and New Zealand position statement: Coronary artery calcium scoring. Heart Lung Circ 2017;26(12):1239-51. doi: 10.1016/j.hlc.2017.05.130.

4. Greenland P, Alpert JS, Beller GA, et al. 2010 ACCF/AHA guideline for assessment of cardiovascular risk in asymptomatic adults: Executive summary: A report of the American
College of Cardiology Foundation/American Heart Association Task Force on practice guidelines. Circulation 2010;122(25):2748-64. doi: 10.1161/ CIR.0b013e3182051bab.

5. Michos ED, Nasir K, Braunstein JB, et al. Framingham risk equation underestimates subclinical atherosclerosis risk in asymptomatic women. Atherosclerosis 2006;184(1):201-06. doi: 10.1016/j.atherosclerosis.2005.04.004.

6. Schlendorf KH, Nasir K, Blumenthal RS. Limitations of the Framingham risk score are now much clearer. Prev Med 2009;48(2):115-16. doi: 10.1016/j.ypmed.2008.12.002.

7. Greenland $\mathrm{P}$, Bonow RO, Brundage BH, et al. ACCF/AHA 2007 clinical expert consensus document on coronary artery calcium scoring by computed tomography in global cardiovascular risk assessment and in evaluation of patients with chest pain. Circulation 2007;115(3):402-26. doi: 10.1161/CIRCULATIONAHA.107.181425.

8. Demer LL, Tintut Y. Vascular calcification: Pathobiology of a multifaceted disease. Circulation 2008;117(22):2938-48. doi: 10.1161/ CIRCULATIONAHA.107.743161.

9. Stary $\mathrm{HC}$. The sequence of cell and matrix changes in atherosclerotic lesions of coronary arteries in the first forty years of life. Eur Heart J 1990;11 Suppl E:3-19. doi: 10.1093/eurheartj/11.suppl_e.3. 
10. Bartel AG, Chen JT, Peter RH, Behar VS, Kong Y, Lester RG. The significance of coronary calcification detected by fluoroscopy. A report of 360 patients. Circulation 1974;49(6):1247-53. doi: 10.1161/01.cir.49.6.1247.

11. Rumberger JA, Simons DB, Fitzpatrick LA, Sheedy PF, Schwartz RS. Coronary artery calcium area by electron-beam computed tomography and coronary atherosclerotic plaque area. A histopathologic correlative study. Circulation 1995;92(8):2157-62. doi: 10.1161/01.cir.92.8.2157.

12. Baumgart D, Schmermund A, Goerge $G$, et al. Comparison of electron beam computed tomography with intracoronary ultrasound and coronary angiography for detection of coronary atherosclerosis. J Am Coll Cardiol 1997;30(1):57-64. doi: 10.1016/s0735-1097(97)00147-2.

13. Voros S, Rivera JJ, Berman DS, et al. Guideline for minimizing radiation exposure during acquisition of coronary artery calcium scans with the use of multidetector computed tomography: A report by the Society for Atherosclerosis Imaging and Prevention Tomographic Imaging and Prevention Councils in collaboration with the Society of Cardiovascular Computed Tomography. J Cardiovasc Comput Tomogr 2011;5(2):75-83. doi: 10.1016/j.jcct.2011.01.003.

14. Agatston AS, Janowitz W, Hildner FJ, Zusmer NR, Viamonte M Jr, Detrano R. Quantification of coronary artery calcium using ultrafast computed tomography. J Am Coll Cardiol 1990;15(4):827-32. doi: 10.1016/0735-1097(90)90282-t.

15. Detrano R, Guerci AD, Carr JJ, et al. Coronary calcium as a predictor of coronary events in four racial or ethnic groups. N Engl J Med 2008;358(13):1336-45. doi: 10.1056/ NEJMoa072100.

16. Mitchell JD, Paisley R, Moon P, Novak E Villines TC. Coronary artery calcium and long-term risk of death, myocardial infarction, and stroke: The Walter Reed cohort study. JACC Cardiovasc Imaging 2018:11(12):1799-806. doi: 10.1016/j. jcmg.2017.09.003.

17. Budoff MJ, Shaw LJ, Liu ST, et al. Long-term prognosis associated with coronary calcification: Observations from a registry of 25,253 patients. J Am Coll Cardiol 2007;49(18):1860-70. doi: 10.1016/j.jacc.2006.10.079.

18. Yano Y, O'Donnell CJ, Kuller L, et al. Association of coronary artery calcium score vs age with cardiovascular risk in older adults: An analysis of pooled population-based studies. JAMA Cardiol 2017;2(9):986-94. doi: 10.1001/ jamacardio.2017.2498

19. Kavousi M, Desai CS, Ayers C, et al. Prevalence and prognostic implications of coronary artery calcification in low-risk women: A meta-analysis. JAMA Cardiol 2016;316(20):2126-34. doi: 10.1001/ jama.2016.17020.

20. Silverman MG, Blaha MJ, Krumholz HM, et al. Impact of coronary artery calcium on coronary heart disease events in individuals at the extremes of traditional risk factor burden: The multi-ethnic study of atherosclerosis. Eur Heart J 2014;35(33):2232-41. doi: 10.1093/eurheartj/ eht508.

21. Sarwar A, Shaw LJ, Shapiro MD, et al. Diagnostic and prognostic value of absence of coronary artery calcification. JACC Cardiovasc Imaging 2009;2(6):675-88. doi: 10.1016/j.jcmg.2008.12.031.

22. Valenti V, Ó Hartaigh B, Heo R, et al. A 15-year warranty period for asymptomatic individuals without coronary artery calcium: A prospective follow-up of 9,715 individuals. JACC Cardiovasc Imaging 2015;8(8):900-09. doi: 10.1016/j. jcmg.2015.01.025
23. Blaha M, Budoff MJ, Shaw LJ, et al. Absence of coronary artery calcification and all-cause mortality. JACC Cardiovasc Imaging 2009;2(6):692-700. doi: 10.1016/j. jcmg.2009.03.009.

24. Nasir K, Bittencourt MS, Blaha MJ, et al. Implications of coronary artery calcium testing among statin candidates according to American College of Cardiology/American Heart Association cholesterol management guidelines: MESA (Multi-Ethnic Study of Atherosclerosis). J Am Coll Cardiol 2015;66(15):1657-68. doi: 10.1016/j. jacc.2015.07.066.

25. Polonsky TS, McClelland RL, Jorgensen NW, et al. Coronary artery calcium score and risk classification for coronary heart disease prediction. JAMA 2010;303(16):1610-16. doi: 10.1001/jama.2010.461.

26. Erbel R, Möhlenkamp S, Moebus S, et al. Coronary risk stratification, discrimination, and reclassification improvement based on quantification of subclinical coronary atherosclerosis: The Heinz Nixdorf recall study. J Am Coll Cardiol 2010:56(17):1397-406. doi: 10.1016/j.jacc.2010.06.030.

27. Elias-Smale SE, Proença RV, Koller MT, et al. Coronary calcium score improves classification of coronary heart disease risk in the elderly: The Rotterdam study. J Am Coll Cardiol 2010;56(17):1407-14. doi: 10.1016/j. jacc.2010.06.029.

28. Okwuosa TM, Greenland P, Ning $\mathrm{H}$, et al. Distribution of coronary artery calcium scores by Framingham 10-year risk strata in the MESA (Multi-Ethnic Study of Atherosclerosis) potential implications for coronary risk assessment. J Am Coll Cardiol 2011;57(18):1838-45. doi: 10.1016/j. jacc.2010.11.053.

29. Knez A, Becker A, Leber A, et al. Relation of coronary calcium scores by electron beam tomography to obstructive disease in 2,115 symptomatic patients. Am J Cardiol 2004;93(9):1150-52. doi: 10.1016/j. amjcard.2004.01.044.

30. Saremi F, Achenbach S. Coronary plaque characterization using CT. AJR Am J Roentgenol 2015;204(3):W249-60. doi: 10.2214/AJR.14.13760.

31. Williams MC, Kwiecinski J, Doris M, et al. Low-attenuation noncalcified plaque on coronary computed tomography angiography predicts myocardial infarction: Results from the multicenter SCOT-HEART trial (Scottish Computed Tomography of the HEART). Circulation 2020:141(18):1452-62. doi: 10.1161/ CIRCULATIONAHA.119.044720.

32. Cho I, Chang HJ, Sung J et al. Coronary computed tomographic angiography and risk of all-cause mortality and nonfatal myocardial infarction in subjects without chest pain syndrome from the CONFIRM registry. Circulation 2012;126(3):304-13. doi: 10.1161/CIRCULATIONAHA.111.081380.

33. Cho I, Al'Aref SJ, Berger A, et al. Prognostic value of coronary computed tomographic angiography findings in asymptomatic individuals: A 6-year follow-up from the prospective multicentre international CONFIRM study. Eur Heart J 2018;39(11):934-41. doi: 10.1093/eurheartj/ehx774.

34. Lee Cl, Tsai EB, Sigal BM et al. Incidental extracardiac findings at voronary CT: Clinical and economic impact. AJR Am J Roentgenol 2010;194(6):1531-38. doi: 10.2214/AJR.09.3587.

35. Miedema MD, Duprez DA, Misialek JR, et al. Use of coronary artery calcium testing to guide aspirin utilization for primary prevention: Estimates from the multi-ethnic study of atherosclerosis. Circ Cardiovasc Qual Outcomes 2014;7(3):453-60. doi: 10.1161/CIRCOUTCOMES.113.000690.
36. Blaha MJ, Budoff MJ, DeFilippis AP, et al. Associations between $\mathrm{C}$-reactive protein coronary artery calcium, and cardiovascular events: Implications for the JUPITER population from MESA, a population-based cohort study. Lancet;378(9792):684-92. doi: 10.1016/S01406736(11)60784-8.

37. Mitchell JD, Fergestrom N, Gage BF, et al. Impact of statins on cardiovascular outcomes following coronary artery calcium scoring. J Am Coll Cardiol 2018;72(25):3233-42. doi: 10.1016/j. jacc.2018.09.051.

38. Budoff MJ, Young R, Lopez VA, et al. Progression of coronary calcium and incident coronary heart disease events: MESA (MultiEthnic Study of Atherosclerosis). J Am Col Cardiol 2013;61(12):1231-39. doi: 10.1016/j. jacc.2012.12.035.

39. Min JK, Lin FY, Gidseg DS, et al. Determinants of coronary calcium conversion among patients with a normal coronary calcium scan: What is the 'warranty period' for remaining normal? J Am Coll Cardiol 2010;55(11):1110-17. doi: 10.1016/j. jacc.2009.08.088.

40. Lee S-E, Chang H-J, Sung JM, et al. Effects of statins on coronary atherosclerotic plaques: The PARADIGM Study. JACC Cardiovasc Imaging 2018;11(10):1475-84. doi: 10.1016/j. jcmg.2018.04.015.

41. Divakaran S, Cheezum MK, Hulten EA, et al. Use of cardiac CT and calcium scoring for detecting coronary plaque: Implications on prognosis and patient management. $\mathrm{Br} J$ Radiol 2015:88(1046):20140594. doi: 10.1259/ bjr.20140594. 